\title{
Underwater Routing Protocols: Analysis of Intrepid Link Selection Mechanism, Challenges and Strategies
}

\author{
Shahzad Ashraf ${ }^{*}$, Zeeshan Aslam², Adnan Yahya ${ }^{3}$, Adnan Tahir \\ ${ }^{1}$ College of Internet of Things Engineering, Hohai University, Changzhou, Jiangsu, China \\ ${ }^{2}$ Petroweld Oilfield Services Kurdistan, Iraq \\ ${ }^{3}$ Dow University of Health Sciences, Karachi Pakistan \\ ${ }^{4}$ College of Software Engineering, Shenzhen University Shenzhen, China \\ *Corresponding Author: nfc.iet@hotmail.com,Tel.: +86-15651229815
}

Available online at: www.isroset.org

Received: 03/Apr/2020, Accepted: 16/Apr/2020, Online: 30/Apr/2020

\begin{abstract}
The sensor nodes deployed in underwater environment have a different routing mechanism in contrast to the terrestrial network. In order to get underwater information from dynamically deployed senor nodes, a smooth packet transmission must be maintained, which is a crucial challenge, and selecting the best communication link between source and destination node is a key phenomenon. The meticulous research has been conducted to search out the best link selection mythology of bodacious underwater routing protocol EnOR, SURS - PES and USPF. The performance has been evaluated through NS2 simulation for packet delivery ratio, end-to-end delay, network lifespan and network energy consumption.
\end{abstract}

$\underline{\text { Keywords - Packet forwarding; relay node; network throughput, underwater routing }}$

\section{INTRODUCTION}

It is a demanding task to develop routing protocols for underwater sensor networks (UWSNs), because they are highly hampered by poor acoustic connections. Traditionally, a UWSN consists of underwater sensor nodes, which are used to sense the atmosphere and unusual activities, and shore sonobuoys (sinks), that are responsible for gathering sensed data from the nodes. Optical and radio frequency interactions in such networks are generally deemed infeasible because optical signals suffer from extreme interference, while high-energy radio signals are easily absorbed due to high attenuation [1]. The acoustic channel was therefore seen as the only feasible system for wireless contact underwater. None the less, this system brings restricted network efficiency, large and intermittent delays, transient loss of path and large noise, multi-path flickering, shadow zones and high energy cost of contact.

Acoustically, the underwater wave operates at $1500 \mathrm{~m} / \mathrm{s}$, which is less than the electromagnetic wave frequency in five order magnitudes [2]. The acoustic channel has a continuum of temporal frequency with spatial underuse. Numerous challenges are faced by UWSN's low bandwidth with absurdly high channel error rate, transient route failure and weak channel multipath, including node displacement 2-3 m/s at water current unlike the terrestrial device [3]. Even while the speed of the acoustic wave is seemingly constant underwater, but due to the unpredictable nature of submarine organisms and water current, as it hits the sea, it reflects in multipurpose directions. Therefore, variations in sound speed are achieved, but a directional transmission can decrease this likelihood.

The multi-hop routing causes packet failure with an expected delay factor contributing to poor communication, so enough re-transmissions are needed to transmit packets reliably. Under these volatile and tough environments, the localized sensor nodes are completely battery-dependent and hard to replace, though replacement will increase high bits [4]. UWSN enhances the dimensions of exploration of underwater warfare to unforeseen and uncertain circumstances, such as ocean collisions, the atmosphere and seismic warnings, the quality of pollutants, situational monitoring, sampling offshore and navigation. The UWSNs have given incredible success in the petroleum and gas business. Oceanographic data, mine identifications, underwater tracking and seafood items may also be collected. A submarine protocol specifies the size of a load and the bit error packet. Not only does the network output degrade, but also wastes resources through inadequate packet size selection [5]. The efficiency of the underwater network depends greatly on topology architecture, which is driven by a selection process for epitome relief nodes that 
increases the likelihood of transmission to the destination node. Intelligent topology has a very smaller energy consumption ratio than an insecure and less efficient topology.

The meticulously findings have been conducted in regards to the communication link selection process thereby considered three state of the art underwater routing protocols and their link establishment process has been analyzed by focusing the methodology which each protocol has adopted and through obtained results in terms of endto-end delay, network throughput and energy consumption. At the end, we came up with best link selection result adopted by one of these protocols. We came across some challenges still faced by the underwater routing and that needs to be considered for future findings.

a. There are two types of routing topologies underwater: Motion dependent topologies, and Coverage dependent topologies. The fixed or localized nodes are followed by a motion-based routing, whereas the coverage-based routing allows UWSN two dimensional and three dimensions [6]. b.

c. Motion based UWSN: Sensor nodes are positioned at defined positions such as, surface buoys or bottom surface to track the other underwater constituent. Such localized sensor nodes had specific fidget characteristics; dynamically and continuously shifting position floats, driven primarily by a navigation device.

d.

e. Coverage based UWSN: It comprises primarily of twodimensional architecture and three-dimensional architecture. The sensor nodes are anchored at the same depth for a two-dimensional topology and use the underwater bridge to contact that is responsible for capturing and transporting raw data to offshore station. The sink nodes are set to a horizontal and a vertical transceiver that captures sensed data from the nodes surrounding it. The sea is $10 \mathrm{~km}$ deep and the vertical transceiver is to be appropriately long, transmitting data to an offshore station while the horizontal transceiver performs the sensory node order for the acquisition of sensation data. The sinks are fitted with acoustic transceiver and wireless transmitter for the control of several parallel communications [7]. Underwater sensor nodes can have a direct connection via a multi-hop path (relay node) to sink node or in-line link. For a sink node far from the sensor node the power needed to send the packet will decrease by a value of over two of the distances [8], the direct link is an easy communication route but not a resource-efficient solution indeed. It also entails a high transmitting capacity and is likely to will the network efficiency and the effect may be significant acoustic interference. A multi-shop communication strategy raises the latency factor as data is exchanged between intermediate nodes in the maze of the routing. Furthermore, in terms of contact ability, two-dimensional underwater routing faces main challenges; selection of the water surface and the size. An improved version of a 2D UWSN is a three-dimensional underwater sensor network; the sensor nodes freely float at arbitrary water level to collect sensed data. Adherence of the sensor nodes at the bottom is a more jingoistic strategy, so buoy retains the sensor nodes so draws into the surface of the water [9]. Depth of anchor nodes may be managed by changing the length of the cable. Some barriers to 3D undersea contact are obstructed, such as the sensor depth should be ingenuously modified to intelligently acquire sensed data and the network topology should be linked.

$f$.

The article is structured as follows. The section II describes process of acoustic signal propagation. Section III addresses connection collection methodologies. Results and debate are summarized in section IV, and open challenges and conclusions were outlined in section $\mathrm{V}$ and VI respectively.

\section{UNDERWATER ACOUSTIC SIGNAL PROPAGATION MECHANISM}

A highly dynamic undersea environment and acoustic communication is often subject to variable factors which require an acoustic channel's bandwidth to remain and to only stay on the frequency and distance of a sensor node. The communicating underwater varies shallow and deep by ocean division. Slightly shallow water had a high temperature, a multipath effect, a surface disturbance, and a broad pause in propagation that eventually adversely affected the output of sound signals; whereas a deep-water sea had the same history with specific measurements [10]. Table 1 describes the main features of shallow and deep water.

Table 1. Shallow v / s Deep water characteristics

\begin{tabular}{lllll}
\hline Features & Shallow Water & Deep Water & \\
\hline Temperature & High & Low & \\
\hline Depth & $\begin{array}{l}\text { Between } 0 \text { and } \\
100 \text { meters }\end{array}$ & & \\
& Surface Reflection & $\begin{array}{l}\text { Reflection of surface and } \\
\text { ground }\end{array}$ & \\
Multi-path loss & Spherical & \\
Spreading & Cylindrical & Sactor & &
\end{tabular}

Several malicious dissemination components are evaluated in sequence as pragmatically. 


\section{A. Factors affecting underwater Acoustic Communication}

Underwater environments are defined as major propagation factors that influence acoustic communication:

i.) Path loss: the diffusion effect indirectly raises the underwater temperature resulting in vacillated movement between the sensor nodes and signal power. The lack of direction is further split into three parts.

a. Geometric Spanning Error: the sound wave is distant but unchanged from frequency [11], where spherical spreading losses rise in deep water, creating a cylindrical lack of stretching in low water.

b. Signal Attenuation: Attenuation is based on frequency and distance between the nodes [12]. That is since acoustic energy is transformed into another form of energy such as heat energy.

c. Dispersion: This happens by shifting angles of acoustic waves. Varying wind speeds cause surface roughness to increase the decline of the dispersion barrier, ultimately causing a delay in transmission and loss of power in subsequent communications [13].

ii.) High propagated delay: When the acoustic signal acts at $1500 \mathrm{~m} / \mathrm{s}$, the transmission has an endless delay factor of approximately $0.67 \mathrm{~s} / \mathrm{km}$, leading to a high propagated layoff. iii.) Noise Ratio: Any inevitable signal intensity condition atrophy forces the device to incorporate a noise ratio. Ambient noise exists in the case of UWSN because of multiple unidentifiable unknown sources. There are four types of ambient noises: (i) wind noise; (ii) noise; (iii) thermal noise. A wind noise created by the varying wind speeds causing acoustic waves to break down. Owing to the acoustic wave interference that is induced by the shipping voyage produces acoustic signals hurdles. Sea surge induces small frequency vibration, which creates noise during communication. A machine also generates a suitable noise, called an auto-noise, that doesn't mimic other sounds, it has a similar relation to the thermal noise level.

iv.) Multipath Tangle: Multipath enigma: Multipath are generated in underwater communication where sound waves impact the water surface and the depths of the ocean causing incoherent acoustic communication interference, resulting in erroneous signal and a multipath effect [15]. An acoustic channel impulse response leaves the variable propagation and power effect dissident. Due to uneven sound speed, many paths are created, and only limited reflection is taken into account and the loss of energy is taken into account.

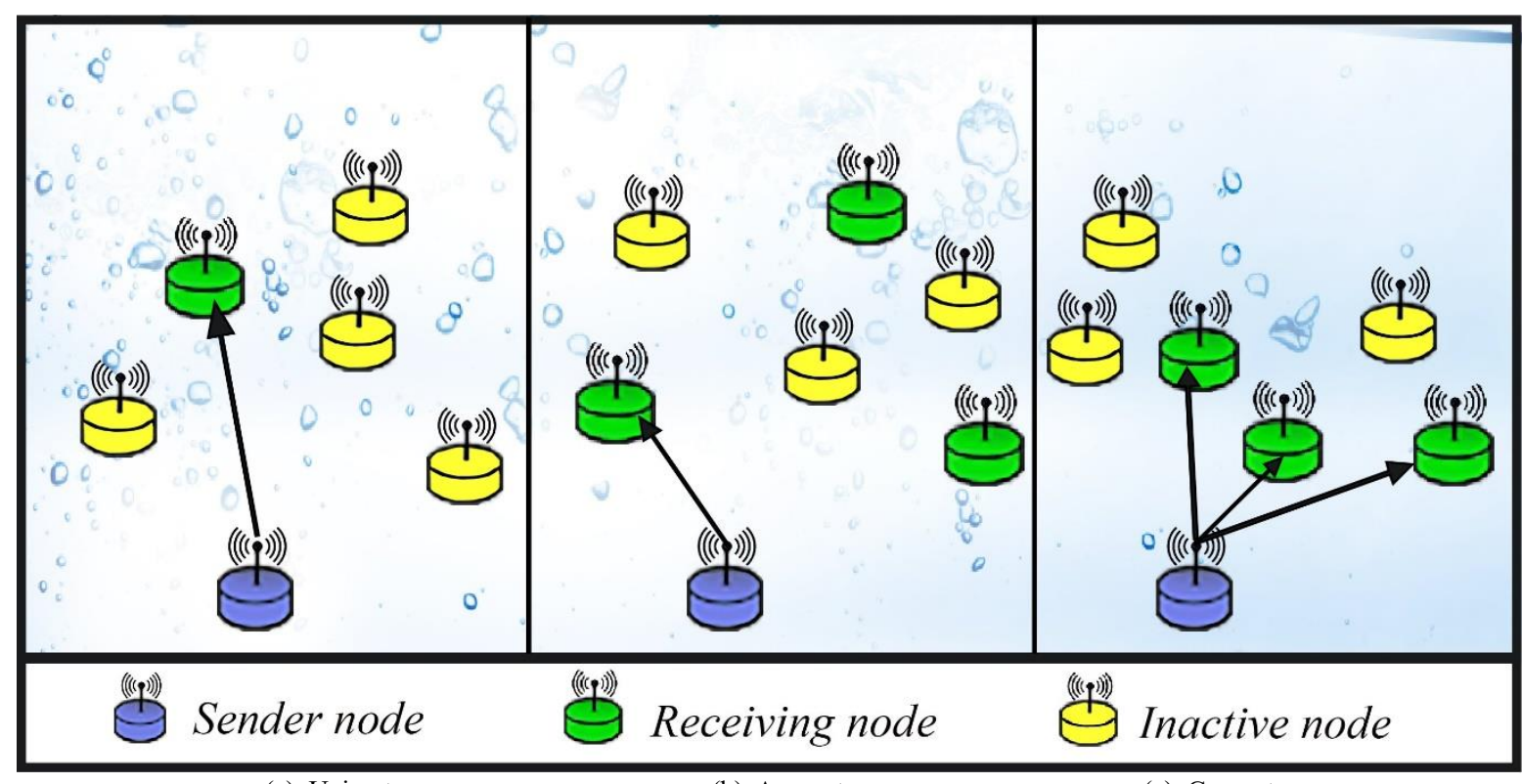

(a) Unicast

(b) Anycast

(c) Geocast

Figure. 1. Various transmission routing schemes

The geographical dynamic routing can be separated in three prominent categories for UWSNs, according to the number and location of destinations, namely unicast, anycast, and geocast indeed as illustrated in Figure1. For a Unicast packet transmission Figure. 2(a), the data is being transmitted from sender node to the single receiving node. The Inactive nodes on the network are not involved in the communication. While Anycast transmission Figure. 2(b), has different routes to two or more endpoint destinations has a common target address. The desired path is chosen based on the number of hops, size, low cost, latency measurements or the least congested route. The Geocast transmission Figure. 2(c), delivers the information to a number of destinations in network classified by geographical locations. A comparative analysis among these transmission routings are placed in Table 2 . 
Generally, all underwater routing approaches have local and global considerations whereas a global approach requires a high overhead computation relative to the local one, and the source node in the network thereby preserved [16]. Moreover, picking of next forwarding node is typically accomplished by dividing the opportunistic routing into two classes: location-base and location-free routing. The location dependent routing picks the forwarding node by taking into account the distance or depth metric up to the node.

\section{LINK SELECTION METHODOLOGIES}

The link selection methodologies of three prominent underwater routing protocols are extensively being investigated through their network architecture and then performance has been analyzed in regards of packet delivery ratio, end-to-end delay, network lifespan and network energy consumption. The findings are discussed as under.

\section{A. Energy Balancing Routing Protocol for Underwater} Sensor Networks (EnOR): Rodolfo W et al [16], discussed one of the immutability problems relating to the priority level of transmission of nodes., resulting in balanced power usage and extended lifespan of UWSN network. It rotates the priority level of transmission for the transmission nodes, taking into account the remaining capacity, reliability of the connection and progression of packets.

Link Selection methodology: A beacon packet is periodically transmitted by each underwater sensor node. The lightning packet comprises the identity of the sender, the remaining information on its energy and its size. Algorithm 1. provides the procedure for selecting the best connection.

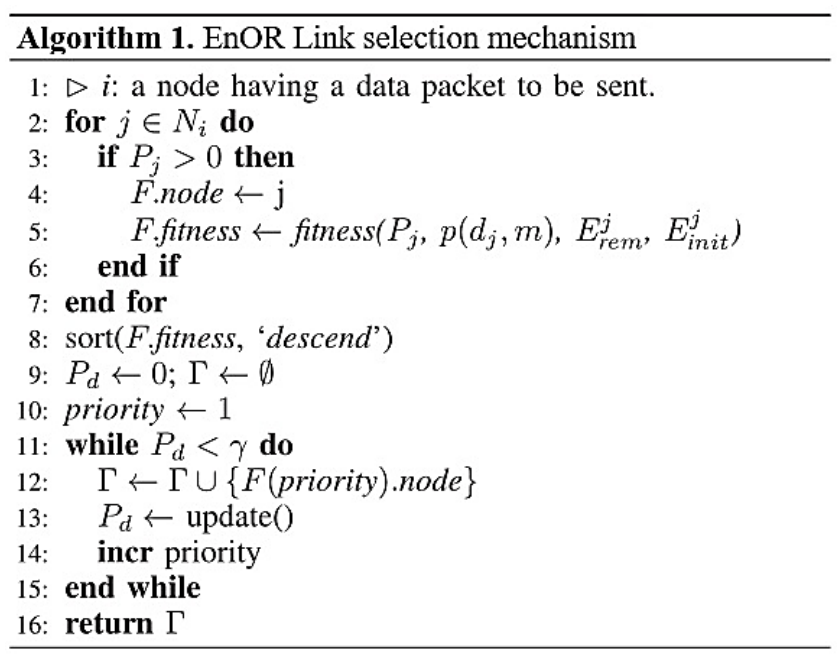

Using $i$ as a sensor node with a data packet to be sent while maintain the neighbouring table as $N i$. The node analyses its adjacent nodes to choose the most suitable nodes to be forwarded (lines 2-7). For this function, only if a neighboring node advances towards the surface sonobuoys can a candidate node be considered. (Lines 3-6). A difference between the current I node sender depth and $j$ in $P_{j}=\operatorname{depth}(i)-\operatorname{depth}(j)$ is used to measure a packet advancement for a neighbor $j$. The fitness of the neighbor $j$ is then determined (line 5). Using connection reliability, packet progress and remaining energy to assess the suitability of a nearby node. This is measured in line 5 and thus as Eq. (1).

$$
F_{j}=P_{j} \times p\left(d_{j}, m\right) \times\left(\frac{E_{r e m}^{j}}{E_{\text {init }}^{j}}\right)
$$

where $P_{j}>0$ represents the packet advancement of node $j$; $p\left(d_{j}, m\right)$ is an estimation of data packet of $m$ between node $i$ and node $j ;{ }_{j}{ }_{\text {rem }}$ shows remaining potency of $j$; and $E_{j_{\text {init }}}$ is the initial value of the potency of $j$.

The nodes allowed the connection to be selected and sorted by fitness value (line 8 ). Finally, from the potential nodes the relation set is calculated. A limited link set can lead to low reliability of the link. At the other side, a wide connecting set may also damage the query, because it takes a long time. The possible node connections are applied to the whole collection until the required link reliability $\gamma$ is reached.

\section{B. Design of Shrewd Underwater Routing Synergy}

Using Porous Energy Shells (SURS-PES) [17]: To transmit the data packet from source to sink-node, the authors used a newly-developed link with residual energy and depth detail. In an area where energy usage has direct impact as the entire active underwater nodes rely on batteries and when cost-effective data packets are delivered, no charge or replacement steps are taken becomes a crucial factor. The authors utilized a shrewd link selection mechanism, if a link is less than or equivalent to $50 \%$ shaky, after broadcasting of a sensor node the destination node is checked, and the destination node is returned to the source node, adding some unusable porous energy shell to strengthen a link from $5 \%$ to full 90 , and then transmitting it to the target.

Link Selection methodology: The link quality inspection has been taken through link reparation mechanism that is depicted in Figure 2. sensor node, $a$, broadcasts the packet, $p$, with substantial information such as depth, ID, and residual energy towards neighboring nodes, i.e., $b, c$, and $d$. The source node, $\mathrm{Na}$ is broadcasting the packet towards neighbors, upon receiving this packet node $b$ includes the necessary information and sends it back as $N b p$ ' to node $a$. 


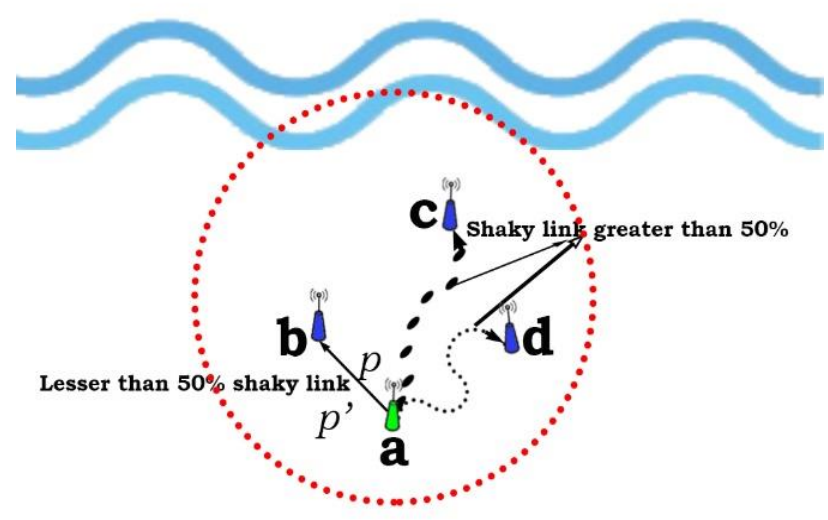

Figure 2. SURS-PES protocol link selection mechanism

When a duplicate node $a$ is attached to the required energy shells, the packet multizes again to node $b$ as $N a 2 p$, in a trivial time $t$, The grain of the final relation is measured as shown in Eq. (2).

$$
\text { Link Grain }=N a_{p}+N b_{p}+N a_{2 p} \text {, }
$$

eventually, the optimal link is being obtained holding energy utilization $E a_{p}, E b_{p}$ and $E a_{2 p}$ respectively thereby remains unchanged thereupon Eq. (3), The probability of connection status from 50 to 90 percent updates in due course.

Link Grain $=t\left(\sum_{p=1}^{N a_{p}} E a_{p}+\sum_{p^{\prime}=1}^{N b_{p \prime}} E b_{p^{\prime}}+\sum_{2 p=1}^{N a_{2 p}} E a_{2 p}\right)$,

There is an exhaustive study of the contact connections between node a and others. Therefore, there is a stipulated connection quality control, which records the hop links are hit by more than $50 \%$ and which links are more stable than $50 \%$ at all. Unlike the consistency of the connection between the source node $a$ and $b$, the connections to the node $a$ and $d$ are more than 50 percent stable, but not up to 90 percent stable. The suggested approach (SURS-PES) therefore takes account of the hop connection between node $a$ or $b$ for the more secure packet transmission, i.e. up to 90 percent.

C. USPF: Underwater shrewd packet flooding mechanism through surrogate holding time

The authors [18] developed a shrewd data forwarding mechanism by taking three unique steps in regards to link selection and packet holding time namely called surrogate holding time. They implemented an angled approach in order to boost the distribution of data packets and to revitalize the life of the network. No single process consists of three stages, from source to sink. Forwarder Hop Angle (FHA) and Counterpart Hop Angle (CHA) are litigated for inclusion of data packets in the first phase of the same transmission field. If a value of FHA is equal to or higher than CHA, the packet produced will be in the same zone of transmission otherwise it would claim that the packet has another maverick. The next step is selecting the best relayscale node by again using the Additive-Rise and AdditiveFall method in three state connection consistency with prefix values. Ultimately, the third stage offers the exorbitant overhead fistula a definitive solution; the package holding time is built to avoid the risk of a packet loss.

Link Selection methodology: The link quality of forwarding node considering $P$ and the neighbor nodes has been explored using Additive-Rise and Additive-Fall methods [33] that shrewdly makes the adjustment to the states of the Forwarding hop angle values as illustrated in Figure 3.

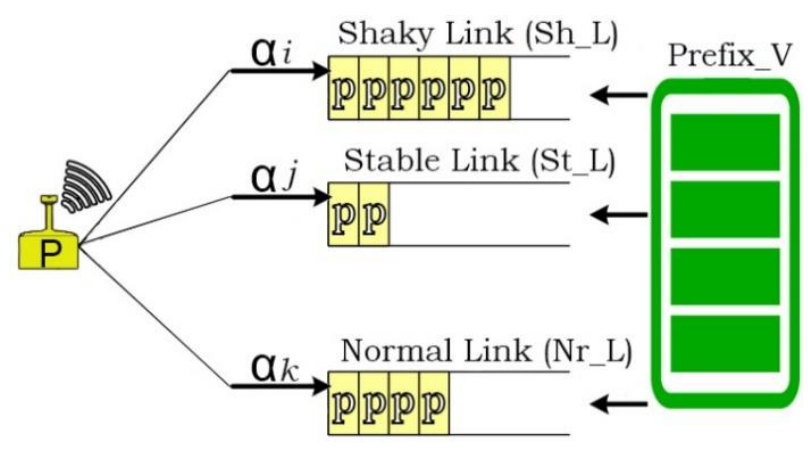

Figure 3. USPF protocol link selection mechanism

The aforementioned three steps are described as below:

Step 1: The forwarder node $p$ changes the route by producing more $\alpha i$ packets to explore more sparsely when the Connection status $\left(S h \_L\right)$ is shaky or slanting compared to the prefix value (Prefix_v) with next nodes.

Step 2: If the connection state $\left(S t_{-} L\right)$ is secure and hence meets the prefix property (Prefix_v), packet forwarding takes place without any obstacles.

Step 3: At a time if the connection state $\left(N r_{-} L\right)$ is regular, but not ready for transmission due to certain salinity consequences, certain energy packets with additional shell have to go ahead and, for this reason, only fewer nodes are involved in transfers.

With relation quality only from forwarder to neighboring nodes, the flood zone is modified. Eq. (4) points,

$F H A p= \begin{cases}\alpha i+s h_{-} L, & \text { if } \alpha i<\text { Prefix_v } \\ \alpha j, & \text { if } \alpha i=\text { Prefix_v } \\ \alpha k+\left(N r_{L}\right)-\left(s_{-} L\right), & \text { if } \alpha i>\text { Prefix_v }\end{cases}$

increasing node temporally updates the threshold value. A stronger connectivity also offers a slight delay. Throughout the reparation of the hop angle of the counterpart, a flood zone is never impacted by a nullity, since the hop angle is complex in hop by hop form. However, any relay node is aware of the hop angle of counterparts in the nodes around, 
which seem to preclude nodes from engaging in the forwarding process.

\section{RESULTS AND DISCUSSION}

After thorough discussion regarding link selection procedure by the aforementioned underwater routing protocols. The results in a packet delivery ratio, end-to-end delay, and network throughput are being analyzed by conducting NS2 simulation sessions. The different sensor nodes between 100 to 400 have been deployed in the 3D region with $800 \mathrm{~m} \times 800 \mathrm{~m} \times 800 \mathrm{~m}$ dimensions and the rest of the simulation parameters are given in Table 3 .

Table 3. Simulation Network parameters

\begin{tabular}{ll}
\hline Parameters & Setting \\
\hline Sensor node area occupied & $800 \mathrm{~m} \times 800 \times 800 \mathrm{~m}^{3}$ \\
\hline Medium & Acoustic \\
Nodes quantity & $100-400$ \\
Nodes initial energy & $98 \mathrm{~J}$ \\
Depth threshold & $8 \mathrm{~m}$ \\
Min: and Max: communication Range & $223 \mathrm{~m}, 245 \mathrm{~m}$ \\
Packet Size & $64 \mathrm{bytes}$ \\
Packet generation frequency & $0.03 \mathrm{pkts} / \mathrm{min}$ \\
Velocity & $1500 \mathrm{~m} / \mathrm{s}$ \\
Node displacement & $0-4 \mathrm{~m} / \mathrm{s}$ \\
Channel capacity & $10 \mathrm{Kbps}$ \\
Frequency channel & $24-28 \mathrm{kHz}$ \\
Transmission power & $1.8 \mathrm{~W} ; 0.75 \mathrm{~W} ; 8 \mathrm{~mW}$ \\
Data packet interval & $48 \mathrm{~s}$ \\
Channel bitrate & $140 \mathrm{~b} / \mathrm{s}$ \\
SNR for Signal Acquisition & $18 \mathrm{~dB}$ \\
Number of rounds taken for simulation & $500 \mathrm{rounds}$ \\
Number of Concentric Circular Rings & 7
\end{tabular}

\section{A. Packet delivery ratio:}

A number arrangement of distributed packets is regarded as the packet delivery ratio as it is obtained at the final destination, i.e. sinks node of some shape or quantity. The average ratio of packets acknowledgment by sink node of all protocols i.e., EnOR, SURS-PES and USPF are being analyzed though Figure 4.

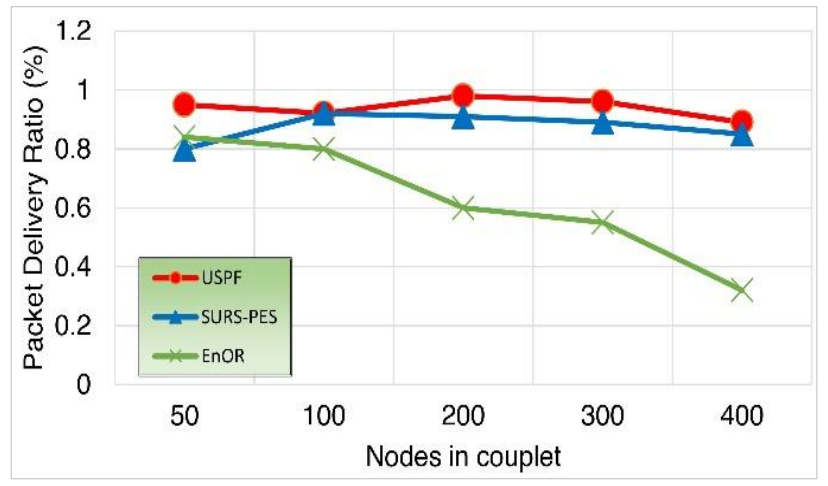

Figure 4. Data packet delivery ratio v/s number of nodes

For EnOR, the delivery ratio is calculated as the fraction of the received packets during transmission cycle and based on the duration of the package distribution percentage reduces. This decrease has taken place since the period decreases by the reduction of live nodes. Comparing with SURS-PES, as the number of nodes are increasing the shrewd packet turnout has achieved. This is due to scrumptious link selection criteria and greater residual energy. Near about 100 to 350 nodes,it achieved a smooth delivery ratio than EnOR approximately $21 \%$ better. Finally, observing USPF performance, by surrogating the $N r_{-} L$ and $S h_{-} L$ values which eventually altered the $S h_{-} L$ angle from 0 to $30^{\circ}$ and $N r_{-} L 0$ to $45^{\circ}$ thereupon, due to changing size of flooding zone in lieu of packet delivery, it came up with unprecedented performance which is about linear from start to the end. Therefore, comparing performance in terms of packet delivery ration with other protocols, USPF gave pristine result.

B. End-to-end delay: The average amount of time is defined as the end-to-end interval for all data packets obtained with performance in the sink node. For all protocols, the overall end-to-end delay has been reported to be decreased with the increasing number of nodes. Infact, this happens as the source seeks more eligible nodes that can forward packets to the corroborated surface sink as depicted in Figure 5.

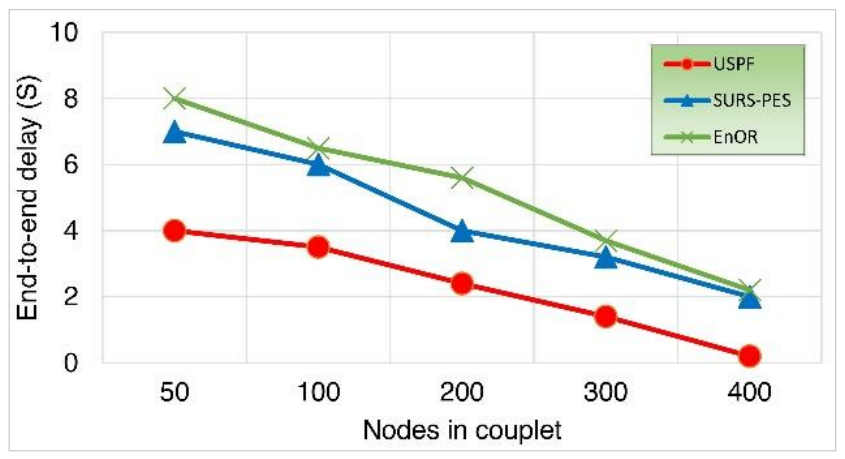

Figure 5. End to end data packet delivery ratio v/s number of nodes

It can be observed that EnOR still has a large end-to-end delay as compare to others because due to fixing of high priority for rotating transmission relay nodes, in addition because of packet holding duration that depends on the nodes priority thereby it has added the greatest gape among sender and the sink node, that is why this delay approximately remained larger than even to the SURSPES. While considering SURS-PES performance, although its performance well and reaching by 200 nodes the delay was drastically lowered while other times it remained linear and this condition remained same in sparse and dense environment, in light of still endless transmission cycle estimates. While considering USPF, once again superb performance can be seen. The USPF has managed minimum end-to-end delays and the highest of all 
due to the shrewd void prevention system. It can be shown that a node needs just 0.3 seconds to manage data from receiving to transmitting systems, meaning that every relay has been inducted for every transmission cycle for at least 0.3 seconds.

C. Network Lifespan: The total amount of time when nodes remain operational in the network is known as network lifespan. The average network lifespan was measured for EnOR, SURS-PES and USPF protocol, as shown in Figure 6.

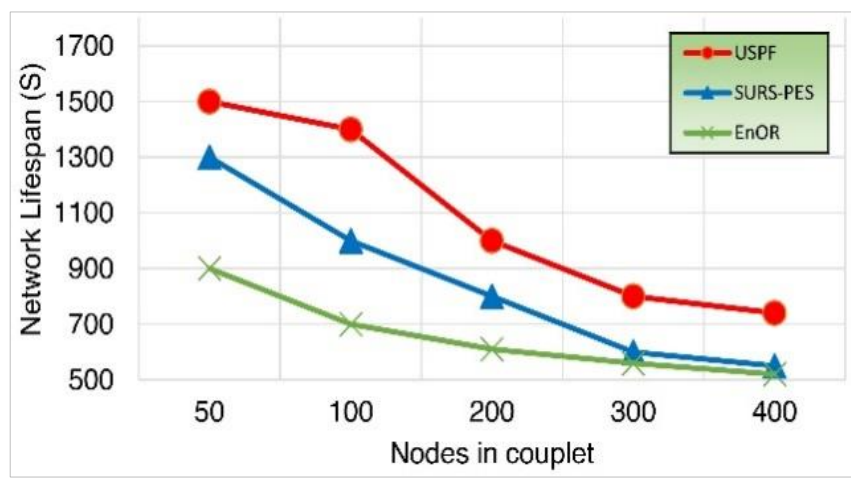

Figure 6. Overall network operational duration

Making judgment about EnOR, it should be noted that, because of its energy costs related to the reception of a packet and the existence of the transmission of subsea acoustic communication which causes it to be received from all its neighbors, even though it is addressed to a specific node the active node is quickly decreased by a high network density. While SURS-PES protocol outperformed as compared EnOR because increased residual energy with shrewd connection factor allowed the network lifetime to stretch throughout transmission. Moreover, it holds no packet, keeping tangle, and thus, no smooth movement of the packet is avoided, no matter how much the network capacity is thick or spacious. Subsequently, the network life under the EnOR and SURSPES protocols are naturally smaller than in the USPF system. It is due to countless reasons like residual energy has been considered when choosing best forwarding relay node and the link selection mechanism with $F H A$ and $C H A$ altering angles preventing the void fistula and thereby no energy wastage has been reported which eventually broaden the network lifespan.

D. Network Energy consumption: An average amount of energy used for full packet transmission from source to the sink node is called Network energy consumption. The simulation result shows the statistics in Figure 7. about the total energy usage by number of nodes for each protocol USPF uses negligible resources to achieve packets to the surface sink node, because it selects the shrewd communication link based on additive rise and fall method in each packet transmission and the holding time of each forwarding relay node prevents packet collisions and retransmissions. While SURS-PES has taken the residual energy and link quality into account for making confine use of energy, but cannot use this strategy for all times, thereon, this condition initially confronted SURS-PES. The energy consumption across the entire $\mathrm{v} / \mathrm{s}$ network of nodes was therefore, marginally higher than before the transfer was done and the energy use ratio could be regulated.

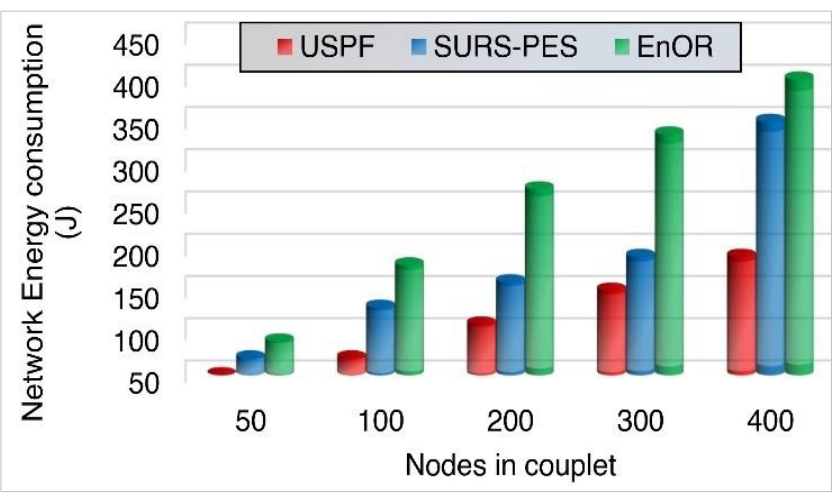

Figure 7. Energy utilization by entire network v/s number of nodes

Considering EnOR protocol, it utilized the rotating transmission priority levels, which engulfed maximum energy reaching by 300 nodes and showed worse performance.

Considering the overall performance by all protocols in terms of the packet delivery ratio, end-to-end delay, network lifespan and network energy consumption it can be ratified that USPF gave outstanding results in all situations.

\section{OPEN CHALLENGS}

The extensive findings regarding communication link selection mythologies and its impact on various underwater routing factors have been presented which unveiled numerous hidden aspects of this amazing field and many open challenges are still need to address and left behind out of which some are highlighted as-

- The noise and reduction channel models are methodological in UWSNs. This field is already available for theoretical or numerical research and study of new models. Furthermore, prototype models may be built to represent the characteristics of the medium underwater.

- With water flows, sensor nodes shift, continuously shifting their locations $[19,20]$. This often shifts their 
place details. It is a challenging task in finding the sensor nodes. The node movement often includes frequent new location changes. This causes uncertainty and energy usage, since these knowledges have to be shared at nodes.

- The UWSN routing protocols use the network layer to supply data packets with water from below. The protocols listed in this survey consider network layer. The network layer may be combined with the MAC layer to reduce packet waiting time in sensor node queues to increase network performance. That would therefore reduce disturbance when sending packets and hence the energy usage. That is because the intrusion contributes to the destruction of packets, and missing packets are recycled, so extra energy is absorbed.

\section{CONCLuSION}

The objective of reviewing communication link selection methodologies by underwear state of the art protocols EnOR, SURS-PES and USPF is to overcome the data routing challenges. It is essential to enhance the link selection mechanism for better and maximum packet delivery yield. The EnOR protocol uses the spinning transfer priority stage uses the available capacity, stability and advancement of the packet to the forwarding relay nodes. While SURS-PES is utilizing a resurrect link factor with residual energy and depth data for the selection of best link and thus forward the packet to the sink node from the source node. The USPF introduced a new technique for link selection mechanism which based on directional flooding by considering two angles, namely Forwarder Hop Angle (FHA) and Counterpart Hop Angle (CHA) with special packet holding time. The performance has been evaluated in relation to packet delivery ratio, end-to-end delay, network lifespan and network energy consumption through the NS2 simulator. The entire finding has revealed that USPF is remained best during all transmission rounds and it performed outstandingly in all stages.

\section{REFERENCES}

[1] Su, Y., Fan, R., Fu, X., \& Jin, Z. (2019). DQELR: An Adazasptive Deep Q-Network-Based Energy- and LatencyAware Routing Protocol Design for Underwater Acoustic Sensor Networks. IEEE Access, 7, 9091-9104. doi:10.1109/access.2019.2891590

[2] S, Ashraf, Gao, M., Chen, Z., Kamran, S., \& Raza, Z. (2017). Efficient Node Monitoring Mechanism in WSN using Contikimac Protocol. International Journal of Advanced Computer Science and Applications, 8(11). doi:10.14569/ijacsa.2017.081152

[3] Qu, J., Zhang, Z., Cui, Y., Wang, J., \& Mastorakis, G. (2019). Research and Application of Multi-Node Communication and
Energy Consumption Prediction Control in Underwater Acoustic Network. IEEE Access, 7, 41220-41229. doi:10.1109/access.2019.2907376

[4] Jin, Z., Zhao, Q., \& Luo, Y. (2020). Routing Void Prediction and Repairing in AUV-Assisted Underwater Acoustic Sensor Networks. IEEE Access doi:10.1109/access.2020.2980043

[5] Tilwari, V., Maheswar, R., Jayarajan, P., Sundararajan, T. V. P., Hindia, M. N., Dimyati, K., Amiri, I. S. (2020). MCLMR: A Multicriteria Based Multipath Routing in the Mobile Ad Hoc Networks. Wireless Personal Communications. doi:10.1007/s11277-020-07159-8

[6] Somani, Arun K., et al. Smart Systems and IoT: Innovations in Computing: Proceeding of SSIC 2019. Springer, 2020.

[7] Coutinho, R. W. L., Boukerche, A., Vieira, L. F. M., \& Loureiro, A. A. F. (2018). Underwater Wireless Sensor Networks. ACM Computing Surveys, 51(1), 1-36. doi: $10.1145 / 3154834$

[8] Dubey, A., \& Rajawat, A. (2016). Impulse effect of node mobility on delay sensitive routing algorithm in underwater sensor network. 2016 International Conference on Internet of Things and Applications (IOTA. doi:10.1109/iota.2016.7562768

[9] Qian, L., Zhang, S., Liu, M., \& Zhang, Q. (2016). A MACAbased power control MAC protocol for Underwater Wireless Sensor Networks. 2016 IEEE/OES China Ocean Acoustics (COA). doi:10.1109/coa.2016.7535810

[10] Cao, J., Dou, J., \& Dong, S. (2015). Balance Transmission Mechanism in Underwater Acoustic Sensor Networks. International Journal of Distributed Sensor Networks, 11(3), 429340. doi:10.1155/2015/429340

[11] Suparti Koul, \& Harmanjot Kour. (2016). Power Efficient Routing in Wireless Sensor Networks. Unpublished. doi:10.13140/RG.2.2.13755.67363

[12] Balsamo, S., Marin, A., \& Vicario, E. (Eds.). (2018). New Frontiers in Quantitative Methods in Informatics. Communications in Computer and Information Science. Springer International Publishing. doi:10.1007/978-3-31991632-3

[13] Balas, Valentina Emilia., et al. Data Management, Analytics and Innovation: Proceedings of ICDMAI 2018, Volume 1. Springer Singapore, 2019.

[14] Liu, X., Liu, P., Long, T., Lv, Z., \& Tang, R. (2018). An efficient depth-based forwarding protocol for underwater wireless sensor networks. 2018 IEEE 3rd International Conference on Cloud Computing and Big Data Analysis doi:10.1109/icccbda.2018.8386561

[15] Ali, M., Khan, A., Mahmood, H., \& Bhatti, N. (2019). Cooperative, reliable, and stability-aware routing for underwater wireless sensor networks. International Journal of Distributed Sensor Networks. doi:10.1177/1550147719854249

[16] Coutinho, R. W. L., Boukerche, A., Vieira, L. F. M., \& Loureiro, A. A. F. (2016). Geographic and Opportunistic Routing for Underwater Sensor Networks. IEEE Transactions on Computers, 65(2), 548-561. doi:10.1109/tc.2015.2423677

[17] Ashraf, S., Ahmed, T., Raza, A., \& Naeem, H. (2020). Design of Shrewd Underwater Routing Synergy Using Porous Energy Shells. Smart Cities, 3(1), 74-92. doi:10.3390/smartcities3010005

[18] Ashraf, S., Gao, M., Mingchen, Z., Ahmed, T., Raza, A., \& Naeem, H. (2020). USPF: Underwater Shrewd Packet Flooding Mechanism through Surrogate Holding Time. Wireless Communications and Mobile Computing, 2020, 112.doi:10.1155/2020/9625974

[19] EL-Bakkouchi, A., Bouayad, A., \& Bekkali, M. E. (2019). A hop-by-hop Congestion Control Mechanisms in NDN Networks - A Survey. 2019 7th Mediterranean Congress of 
Telecommunications (CMT). doi:10.1109/cmt.2019.8931405

[20] Kumari, R., \& Nand, P. (2018). Performance Analysis for MANETs using certain realistic mobility models NS-2. International Journal of Scientific Research in Computer Science and Engineering, 6(1), 70-77. doi: $10.26438 /$ ijsrcse/v6i1.7077

\section{Authors Profile}

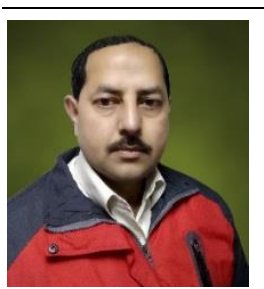

SHAHZAD ASHRAF received B.E. degree in Computer Systems Engineering, and M.E. in Communication System and Networks from Mehran Engineering \& Technology University, Jamshoro Pakistan in 2004, and 2014 respectively. He is currently pursuing the $\mathrm{Ph} . \mathrm{D}$. degree in Information and Communication Engineering with the College of Internet of Things of Engineering, Hohai University Changzhou China. From 2005 to 2016, he served as an Assistant Professor at NFC Institute of Engineering and Technology Multan, Pakistan. He is an active and prominent reviewer of many renowned international journals including IEEE, ACM, wireless personal communications, IET, IETE, international journal of distributed sensor networks, international journal of microwave and wireless technologies, journal of robotics and control, international journal of computers \& technology, and IGI global. His research interests include underwater acoustic sensor network focusing on relay selection and energy harvesting issues, computer hardware and networks, grid and distributed computing, vector graphics and web engineering.

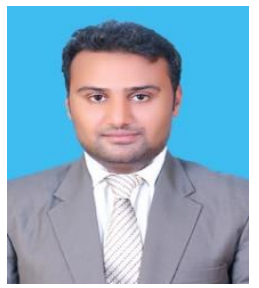

ZEESHAN ASLAM secured B.E. degree in Electrical (Computer System) Engineering from Bahauddin Zakariya University, Multan and an M.S. in Electrical (Power) Engineering from the Institute of Southern Punjab Multan in 2015 and 2018 respectively. Currently, he is working as a HSE site manager, with Petroweld oil field kurdistan, Iraq. From 20152019, he worked as HSE engineer with Volka food international, Multan Pakistan. He was also a visiting lecturer at NFC institute of engineering and technology Multan, Pakistan during 2017-2018.

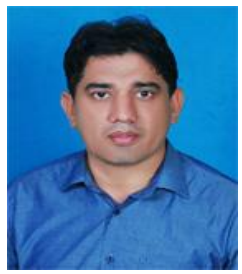

ADNAN YAHYA got B.S in information technology from university of sindh, jamshoro Pakistan and M.S in computer and communication network from Hamdard University Karachi in 2006 and 2019 respectively. He is a certified professional and serving as I.T administrator at DUHS Dow university of health sciences, Karachi

Pakistan and handling overall network design and related deployment issues in a centralized environment with guided and unguided media.

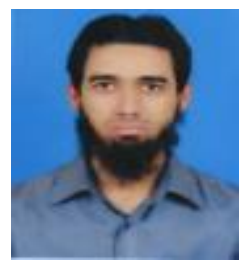

ADNAN TAHIR was awarded masters degree in software engineering specializing in nnformation security from shaheed zulfiqar ali bhutto institute of science \& technology (SZABIST), Islamabad Pakistan in 2014, and secured bachelor degree in computer systems engineering from Bahauddin Zakariya university, multan Pakistan in 2012. Currently he is pursuingtowards his Ph.D. degree in information and communication engineering with college of software engineering, shenzhen university Shenzhen, china. From 2014 to 2016, he served as a lecturer in national college of business administration economics (NCBAE), multan Pakistan. He is an active reviewer of IEEE, ACM, and journal of computer science and technology. His main research interest includes cloud computing, machine learning, fault and error predictions, big data, and information security. 